\title{
DETERMINATION OF AGE AND ORIGINAI CHEMICAL COMPOSITION OF BINARY STARS
}

FERLUGA, S. and ANTILLON, M.

Dipartimento di Astronomia, Università di Trieste Via G.B. Tiepolo, 11 - I 34131 Trieste, Italy

\begin{abstract}
A numerical method is presented, which is able to determine quantitatively the age $t$ and the initial abundances of helium and metals $\left(Y_{0}, Z_{0}\right)$ of a given binary system. Input data are simply the values of mass $m$, temperature $T_{\text {eff }}$ and surface gravity $g$, of both stars. The procedure works for detached main-sequence binaries, and it does not require any preliminary hypothesis about surface abundances or age. It is assumed that the two stars evolve parallely with no interaction.
\end{abstract}

\section{THE PROBLEM}

A star with a given mass $m$, because of evolution, changes its physical properties (as effective temperature $T_{\text {eff }}$ and surface gravity $g$ ), in function of the age $t$, and of the original abundance of helium $Y_{0}$ and metals $Z_{0}$. The relation between observational quantities $\left(m ; \log T_{\text {eff }}, \log g\right)$ and model parameters $\left(Y_{0}, Z_{0} ; t\right)$ is provided by evolutionary theory. Is it then possible to compute composition and age $\left(Y_{0}, Z_{0} ; t\right)$ of a given star, by knowing $\left(m ; \log T_{\text {eff }}, \log g\right)$ from observations?

Here we present an algorithm, which finds out the solution for individual main-sequence binaries. In fact, the job is not possible for single stars, because of practical limitations ( $m$ is unknown), and also because of basic mathematical reasons (equations with infinite solutions). One may wonder why, on the contrary, it is generally possible to solve the problem for a binary system. This may be understood as follows.

At time $t_{0}=0$ (starting main-sequence life), the positions of all stellar masses in the $\left(\log T_{\text {eff }}, \log g\right)$ plane will define the Zams curve; while at time $t_{1}$ they will move sideways on another curve (isochrone $t_{1}$ ), and so on, defining a family of isochrones $t_{i}$. For different compositions $\left(Y_{0}, Z_{0}\right)$, there will be different overlapping families. So, a given star (single mass-point on our plane) may belong to any of the infinite isochrones $t_{i}$ with different $\left(Y_{0}, Z_{0}\right)$, intersecting at that point. Only the presence of a companion star (second masspoint) can individuate the common isochrone, solving the riddle. 


\section{THE BOFER CODE}

Our computer code - called BOFER - performs linear interpolations among a basic grid of stellar models (Mengel et al., 1979), with different initial compositions $\left(Y_{0}=.20, .30 ; Z_{0}=.01, .04\right)$ and ages $\left(t_{i}=0, .07, .1, .2, .5,1,2 \mathrm{~Gy}\right)$, using the corresponding isochrones in the $\left(\log T_{\text {eff }}, \log g\right)$ plane derived by Cester (1982).

Input values are just $\left(m ; \log T_{\text {eff }}, \log g\right)$, with their experimental errors, for the 2 component stars. The range of models, compatible with observational data and experimental errors, is determined by the BOFER code; so, the center of the range and its amplitude define the solution $\left(Y_{0}, Z_{0} ; t\right)$ and its accuracy, respectively.

Binary stars, satisfying our observational requirements, are not numerous: from the published catalogues (Popper, 1980; Andersen, 1991) and from other available material, only two dozens systems can be selected. In fact they should be dwarf, eclipsing systems (giving direct values of $g$ and $m$ ), classified as $d$-type (no mass exchange). They should have non-identical components (2 distinct points on grid) with $\Delta m \geq 0.1 m_{\odot}$, and moreover they must be normal binaries (no triple systems, RS CVn-types, Algols, etc.). Finally, good accuracy is required for the input data: errors on $\left(m ; \log T_{\text {eff }}, \log g\right)$ should not exceed $(3 \% ; 1 \%, 2 \%)$. Actually, admitted masses are from 0.9 to $3.6 \mathrm{~m}_{\odot}$; extension is planned up to $25 m_{\odot}$.

Due to the advanced stage of our computations, the results will become available in the immediate future.

\section{ACKNOWLEDGMENTS}

The initial idea of this method is due to $B$. Cester, who suggested the concept already in 1979. Two years later, the first numerical solutions were presented in a Thesis of one of the authors (Ferluga, 1981). The earliest version of the computer code (BO-FER) was written in collaboration with C. Boehm in 1983.

Financial support for M. Antillon is provided by a grant of the Italian Foreign Ministry.

\section{REFERENCES}

Andersen, J.: 1991, The Astron. Astrophys. Rev. 3, 91

Cester, B.: 1982, private communication

Ferluga, S.: 1981, Helium in Binary Systems (in Italian); Thesis, University of Trieste (Italy)

Mengel, J.G., Sweigart, A.V., Demarque, P., Gross, P.G.: 1979, Ap. J. Suppl. 40, 733

Popper, D.M.: 1980, Ann. Rev. of Astron. and Astrophys. 18, 115. 